\title{
Vibration Analysis of Annular Sector Plates under Different Boundary Conditions
}

\author{
Dongyan Shi, ${ }^{1}$ Xianjie Shi, ${ }^{1}$ Wen L. Li, ${ }^{2}$ Qingshan Wang, ${ }^{1}$ and Jiashan Han ${ }^{3}$ \\ ${ }^{1}$ College of Mechanical and Electrical Engineering, Harbin Engineering University, Harbin 150001, China \\ ${ }^{2}$ Department of Mechanical Engineering, Wayne State University, Detroit, MI 48201, USA \\ ${ }^{3}$ Luoyang Sunrui Special Equipment Co., Ltd., Luoyang 471003, China
}

Correspondence should be addressed to Xianjie Shi; shixianjie@hrbeu.edu.cn

Received 18 October 2013; Accepted 18 May 2014; Published 9 June 2014

Academic Editor: Hassan Haddadpour

Copyright (C) 2014 Dongyan Shi et al. This is an open access article distributed under the Creative Commons Attribution License, which permits unrestricted use, distribution, and reproduction in any medium, provided the original work is properly cited.

\begin{abstract}
An analytical framework is developed for the vibration analysis of annular sector plates with general elastic restraints along each edge of plates. Regardless of boundary conditions, the displacement solution is invariably expressed as a new form of trigonometric expansion with accelerated convergence. The expansion coefficients are treated as the generalized coordinates and determined using the Rayleigh-Ritz technique. This work allows a capability of modeling annular sector plates under a variety of boundary conditions and changing the boundary conditions as easily as modifying the material properties or dimensions of the plates. Of equal importance, the proposed approach is universally applicable to annular sector plates of any inclusion angles up to $2 \pi$. The reliability and accuracy of the current method are adequately validated through numerical examples.
\end{abstract}

\section{Introduction}

Annular sector plates are one of the most important structural components used in industrial applications and civil engineering. The vibrational characteristics of sector plates are thus of great interest to engineers and designers. Although there is a vast pool of studies about vibrations of circular and rectangular plates [1], relatively few results are reported for annular sector plates.

Over the past decades, vibrations of annular sector plates have been investigated using various analytical or numerical methods, such as the energy method [2], spline element method [3, 4], finite element method [5], integral equation method [6], and so on [7]. In particular, a general technique was developed by Leissa [1] to obtain exact modal frequencies for plates which are simply supported along the radial edges and have arbitrary boundary conditions at the circumferential edges. This method utilizes the well-known Bessel function solutions for a circular plate by allowing the functions to have noninteger orders. His following work [8] using Ritz method advocated that the use of the ordinary Bessel functions solution is incorrect for solid sector thin plates having simply supported radial edges and sector angle larger than $\pi$. Liew et al. [9] reviewed many investigations about the vibration of thick plates published before 1993. It is shown that a majority of them are focused on the classical boundary conditions (simply supported, clamped, or free edges). In comparison, other more complicated boundary conditions such as elastic boundary supports are rarely attempted. A closed-form solution is proposed by Kim and Yoo [10] in which the displacements are expressed in terms of trigonometric and exponential functions under the polar coordinate system. Ramakrishnan and Kunukkasseril [11] solved the vibration problem of an annular sector plate with simply supported radial edges and arbitrary conditions along the circumferential edges. Aghdam et al. [12] presented an approximate solution for bending deformation of thin sector plates using extended Kantorovich method in which the fourth-order governing equation is converted into two ordinary differential equations. Employing the $p b-2$ RayleighRitz method, Xiang et al. [13] tackled the vibration problem of annular sector Mindlin plates. Frequency parameters for annular sector plates with different geometry parameters and boundary conditions were presented. Liu and Chen [14] proposed an axisymmetric finite element for axisymmetric vibration analysis of annular and circular plates. Civalek 
and Ülker [15] utilized harmonic differential quadrature (HDQ) method to study the linear bending characteristics of circular plates. Civalek [16] compared the methods of differential quadrature $(D Q)$ and harmonic differential quadrature (HDQ). These methods were utilized for buckling, bending, and free vibrations of thin isotropic plates. Accurate threedimensional elasticity solutions of annular sector plates are presented under arbitrary boundary conditions by Liew et al. [17]. X. Wang and Y. Wang [18] extended the differential quadrature (DQ) method to analyze the free vibration problem of thin sector plates. Irie et al. [19] investigated the free vibrations of ring-shaped polar-orthotropic sector plates using a spline function as an admissible function for the deflection of the plates. In this approach, the flexural transverse deflection of sector plates is expressed as a series of the products of the deflection functions of a sectorial beam and a circular beam that satisfy the similar type of boundary conditions. Three-dimensional vibrations of annular sector plates with various boundary conditions were studied by Zhou et al. [20] using Chebyshev-Ritz method. Also the solutions of annular sector plates with reentrant angle are presented in Zhou et al. [20] investigation. Baferani et al. [21] presented an analytical solution for the free vibration of functionally graded (FG) thin annular sector plates resting on elastic foundations. The plates are considered to be simply supported along radial edges and arbitrarily supported at the circumferential edges. Mirtalaie and Hajabasi [22] studied the free vibration analysis of functionally graded (FG) thin annular sector plates with DQ method.

It appears that the previous investigations on the annular sector plates are mostly limited to classical edge conditions. It is widely believed that an exact analytical solution is only possible for an annular sector plate which is simply supported along, at least, two radial edges. However, a variety of possible boundary conditions such as elastic restraints are usually encountered in many engineering applications $[2,8,23]$. Moreover, the existing solution procedures are often only customized for a specific kind of boundary conditions and thus typically require constant modifications of the trial functions and corresponding solution procedures to adapt to different boundary conditions. As a result, the use of the existing solution procedures will result in very tedious calculations and will be easily inundated with a variety of possible boundary conditions. Therefore, it is important to develop an analytical method which is capable of universally dealing with annular sector plates subjected to different boundary conditions. In addition, the results of annular sector plates with reentrant angle are scarce.

In this paper, an improved Fourier series method (IFSM) previously proposed for the vibration analysis of beams and plates [24-28] is extended to annular sector plates under different boundary conditions, including the general elastic restraints. The displacement solution of the annular sector plate, regardless of boundary conditions, is expressed as a new form of trigonometric expansion with accelerated convergence. The reliability and accuracy of the proposed solution technique are validated extensively through numerical examples.

\section{Theoretical Formulations}

2.1. Basic Equations for an Annular Sector Plate. An annular sector plate (consisted with two radial and two circumferential edges) and the coordinate systems used in this investigation are shown in Figure 1. This plate is of constant thickness $h$, inner radius $a$, outer radius $b$, width $R$ of plate in radial direction, and sector angle $\phi$. The plate geometry and dimensions are defined in a cylindrical coordinate system $(r, \theta, z)$. A local coordinate system $(s, \theta, z)$ is also shown in Figure 1, which will be used in the analysis. The boundary conditions for the bending motion can be generally specified in terms of two kinds of restraining springs (translational and rotational) along each edge, resulting in four sets of distributed springs of arbitrary stiffness values.

The governing differential equation for the free vibration of an annular sector plate is given by

$$
D \nabla_{r}^{2} \nabla_{r}^{2} w(r, \theta)-\rho h \omega^{2} w(r, \theta)=0,
$$

where $\nabla_{r}^{2}=\partial^{2} / \partial r^{2}+\partial / r \partial r+\partial^{2} / r^{2} \partial \theta^{2}, w(r, \theta)$ is the flexural displacement, $\omega$ is angular frequency, and $D=E h^{3} /(12(1-$ $\left.\left.\mu^{2}\right)\right), \rho$, and $h$ are the flexural bending rigidity, the mass density, and the thickness of the plate, respectively.

In terms of the flexural displacement, the bending and twisting moments and transverse shearing forces can be expressed as

$$
\begin{aligned}
M_{r} & =-D\left[\frac{\partial^{2} w}{\partial r^{2}}+\frac{\mu}{r}\left(\frac{\partial w}{\partial r}+\frac{1}{r} \frac{\partial^{2} w}{\partial \theta^{2}}\right)\right], \\
M_{\theta} & =-D\left[\frac{1}{r}\left(\frac{\partial w}{\partial r}+\frac{1}{r} \frac{\partial^{2} w}{\partial \theta^{2}}\right)+\mu \frac{\partial^{2} w}{\partial r^{2}}\right], \\
M_{r \theta} & =-(1-\mu) \frac{D}{r}\left[\frac{\partial^{2} w}{\partial r \partial \theta}-\frac{1}{r} \frac{\partial w}{\partial \theta}\right], \\
Q_{r} & =-D \frac{\partial\left(\nabla^{2} w\right)}{\partial r}, \\
Q_{\theta} & =-D \frac{1}{r} \frac{\partial\left(\nabla^{2} w\right)}{\partial \theta},
\end{aligned}
$$

where $\mu$ is Poisson's ratio.

The boundary conditions for an elastically restrained annular sector plate are given as

$$
\begin{aligned}
& k_{r a} w=Q_{r}, \quad \frac{K_{r a} \partial w}{\partial r}=-M_{r} \quad \text { at } r=a, \\
& k_{r b} w=-Q_{r}, \quad \frac{K_{r b} \partial w}{\partial r}=M_{r} \quad \text { at } r=b \\
& k_{\theta 0} w=Q_{\theta}, \quad \frac{K_{\theta 0} \partial w}{r \partial r}=-M_{\theta} \quad \text { at } \theta=0, \\
& k_{\theta 1} w=-Q_{\theta}, \quad \frac{K_{\theta 1} \partial w}{r \partial r}=M_{\theta} \quad \text { at } \theta=\phi,
\end{aligned}
$$

where $k_{r a}$ and $k_{r b}\left(k_{\theta 0}\right.$ and $\left.k_{\theta 1}\right)$ are translational spring constants, and $K_{r a}$ and $K_{r b}\left(k_{\theta 0}\right.$ and $\left.k_{\theta 1}\right)$ are the rotational 


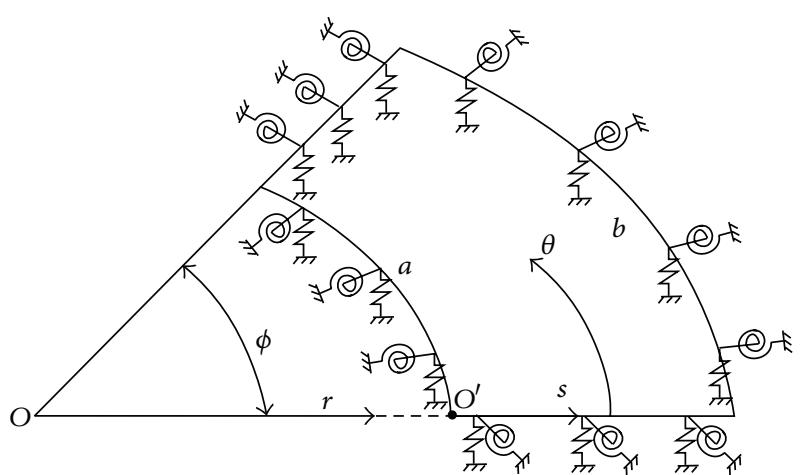

(a)

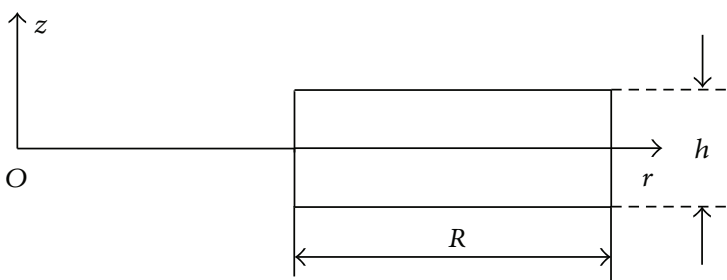

(b)

FIGURE 1: Geometry and dimensions of an annular sector plate. (a) Annular sector plate. (b) Cross section of the annular sector plate.

spring constants at $r=a$ and $b(\theta=0$ and $\phi)$, respectively. All the classical homogeneous boundary conditions can be simply considered as special cases when the spring constants are either extremely large or substantially small. The units for the translational and rotational springs are $\mathrm{N} / \mathrm{m}$ and $\mathrm{Nm} / \mathrm{rad}$, respectively.

The solution for the vibration problem of an annular sector plate can be generally written in the forms of Bessel functions [1]:

$$
\begin{gathered}
w(r, \theta)=\sum_{n=0}^{\infty}\left[A_{n} J_{n}(k r)+B_{n} Y_{n}(k r)+C_{n} I_{n}(k r)\right. \\
\left.+D_{n} K_{n}(k r)\right] \cos n \theta, \\
+\sum_{n=1}^{\infty}\left[A_{n}^{*} J_{n}(k r)+B_{n}^{*} Y_{n}(k r)+C_{n}^{*} I_{n}(k r)\right. \\
\left.+D_{n}^{*} K_{n}(k r)\right] \sin n \theta,
\end{gathered}
$$

where $k r \equiv \lambda, J_{n}$ and $Y_{n}$ are the Bessel functions of the first and second kinds, respectively, and $I_{n}$ and $K_{n}$ are modified Bessel functions of the first and second kinds, respectively. The coefficients, $A_{n}, \ldots, D_{n}, A_{n}^{*}, \ldots, D_{n}^{*}$, which determine the shape of a mode, are to be solved from the boundary conditions.

If the boundary conditions are symmetric with respect to one or more diameters of the plate, the terms involving $\sin n \theta$ are not needed and the solution (4) is simplified to

$$
\begin{gathered}
w(r, \theta)=\sum_{n=0}^{\infty}\left[A_{n} J_{n}(k r)+B_{n} Y_{n}(k r)+C_{n} I_{n}(k r)\right. \\
\left.+D_{n} K_{n}(k r)\right] \cos n \theta .
\end{gathered}
$$

The characteristic equation is derived by substituting the solution into the boundary conditions and setting the determinant of the resulting coefficient matrix equal to zero. The eigenvalues are obtained as the roots of characteristic equation using an appropriate nonlinear root-searching algorithm. The eigenvalues can also be found approximately since the Bessel functions are tabulated in many mathematical books or handbooks. Regardless of what procedures are adopted, the results are understandably dependent on the specific set of boundary conditions involved. The modal properties for annular plates are comprehensively reviewed in [1] for various boundary conditions or complicating factors. However, annular sector plates are rarely dealt with in the literature. This is evident from the fact that reviewing the vibrations of sector plates occupies only less than two pages in the classical monograph [1].

\subsection{An Accelerated Trigonometric Series Representation for the} Displacement Function. In the previous papers [26, 29], each displacement component of a rectangular plate is expressed as a 2D Fourier cosine series supplemented by eight auxiliary terms, which are introduced to accelerate the convergence of the series expansion. In this study, a similar but much simpler and more concise form of series expansion is employed to expand the flexural displacement of an annular sector plate in local coordinate system $(s, \theta, z)$ :

$$
w(s, \theta)=\sum_{m, n=-4}^{\infty} A_{m n} \varphi_{m}(s) \varphi_{n}(\theta) \quad(s=r-a)
$$

where $A_{m n}$ denotes the series expansion coefficients and

$$
\varphi_{m}(s)=\left\{\begin{array}{ll}
\cos \lambda_{m} s & m \geq 0 \\
\sin \lambda_{m} s & m<0
\end{array} \quad\left(\lambda_{m}=\frac{m \pi}{R}\right) .\right.
$$

The basis function $\varphi_{n}(\theta)$ in the $\theta$-direction is also given by (7) except for $\lambda_{n}=n \pi / \phi$. The sine terms in the above equation are introduced to overcome the potential discontinuities, along the edges of the plate, of the displacement function when it is periodically extended and sought in the form of trigonometric series expansion. As a result, the Gibbs effect can be eliminated and the convergence of the series expansion can be substantially improved.

To clarify this point, consider a function $f(x)$ having $C^{n-1}$ continuity on the interval $[0, \pi]$ and the $n$th derivative is absolutely integrable (the $n$th derivative may not exist at 
certain points). Denote the partial sum of the trigonometric series as

$$
\mathrm{F}_{M, 2 P}[f](x)=\sum_{m=-2 P}^{M} a_{m} \varphi_{m}(x) .
$$

It can then be mathematically proven that the series expansion coefficients satisfy

$$
\lim _{m \rightarrow \infty} a_{m} m^{2 P}=0 \quad(\text { for } 2 P \leq n)
$$

if the negatively indexed coefficients, $a_{m}(m<0)$, are calculated from

$$
\begin{gathered}
a_{m}=\sum_{k=1}^{P}\left[(-1)^{m} f^{(2 k-1)}(\pi)+f^{(2 k-1)}(0)\right] \\
\quad \times \frac{\sum_{1 \leq j_{1}<\cdots<j_{P-k} \leq P, j_{1}, \ldots, j_{P-k} \neq i} x_{j_{1}}^{2} \cdots x_{j_{P-k}}^{2}}{x_{i} \prod_{j=1, j \neq i}^{P}\left(x_{j}^{2}-x_{i}^{2}\right)}, \\
m=x_{i}=\left\{\begin{array}{ll}
2 i-1 & \text { if } m \text { is odd } \\
2 i & \text { if } m \text { is even, }
\end{array} \quad(i=1,2, \ldots, P) .\right.
\end{gathered}
$$

More explicitly, the convergence estimate (9) can be expressed as

$$
a_{m}=\mathcal{O}\left(m^{-(2 P+1)}\right) \text { for } 2 P \leq n,
$$

which means

$$
\max _{0 \leq x \leq \pi}\left|f(x)-\mathrm{F}_{M, 2 P}[f](x)\right|=\mathcal{O}\left(M^{-2 P}\right) .
$$

It is seen that convergence can be drastically improved virtually at no extra cost. It should be pointed out that the convergence rate of the series expansion (8) can be controlled by setting $P$ to any appropriate value. In reality, however, the smoothness of the solution required for a given boundary value problem is mathematically dictated by the highest order of derivatives that appeared in the governing differential equation. Take the current plate problem for example. The plate equation demands that the third-order derivatives are continuous and the fourth-order derivatives exist everywhere over the surface area of the plate. Accordingly, one needs to set $P=2$ in seeking for a strong $C^{3}$ solution, or $P=1$ for $C^{1}$ solution in a weak formulation. Because the smoothness (or, explicitly, the convergence rate) of the current series expansion can be managed, at will, over the solution domain, the unknown series expansion coefficients can be obtained from either a weak or strong formulation. In seeking for a strong form of solution, the series is required to simultaneously satisfy the governing equation and the boundary conditions exactly on a pointwise basis. As a consequence, the expansion coefficients are not totally independent; the negatively indexed coefficients are related to the others via the boundary conditions. In a weak formulation such as the Rayleigh-Ritz technique, however, all the expansion coefficients are considered as the generalized coordinates independent from each other.
The strong and weak solutions are mathematically equivalent if they are constructed with the same degree of smoothness over the solution domain. The Rayleigh-Ritz technique will be adopted in this study since the solution can be obtained much easily. More importantly, such a solution process is more suitable for future modeling of built-up structures.

2.3. Final System for an Annular Sector Plate. For a purely bending plate, the total potential energy can be expressed as

$V_{p}$

$$
\begin{aligned}
=\frac{D}{2} \int_{0}^{\phi} \int_{0}^{R} & {\left[\left(\frac{\partial^{2} w}{\partial s^{2}}+\frac{1}{s+a} \frac{\partial w}{\partial s}+\frac{1}{(s+a)^{2}} \frac{\partial^{2} w}{\partial \theta^{2}}\right)^{2}\right.} \\
& -2(1-\mu) \frac{\partial^{2} w}{\partial s^{2}}\left(\frac{1}{s+a} \frac{\partial w}{\partial s}+\frac{1}{(s+a)^{2}} \frac{\partial^{2} w}{\partial \theta^{2}}\right) \\
& \left.+2(1-\mu)\left\{\frac{\partial}{\partial s}\left(\frac{1}{s+a} \frac{\partial w}{\partial \theta}\right)^{2}\right\}^{2}\right](s+a) d s d \theta
\end{aligned}
$$

By neglecting rotary inertia, the kinetic energy of the annular sector plate is given by

$$
T=\frac{1}{2} \rho h \omega^{2} \int_{0}^{R} \int_{0}^{\phi} w^{2}(s+a) d s d \theta .
$$

The potential energies stored in the boundary springs are calculated as

$$
\begin{aligned}
V_{s}=\frac{1}{2} \int_{0}^{\phi}\left\{a\left[k_{r a} w^{2}+K_{r a}\left(\frac{\partial w}{\partial s}\right)^{2}\right]_{s=0}\right. \\
\left.+b\left[k_{r b} w^{2}+K_{r b}\left(\frac{\partial w}{\partial s}\right)^{2}\right]_{s=R}\right\} d \theta \\
+\frac{1}{2} \int_{0}^{R}\left\{\left[k_{\theta 0} w^{2}+K_{\theta 0}\left(\frac{\partial w}{(s+a) \partial \theta}\right)^{2}\right]_{\theta=0}\right. \\
\left.+\left[k_{\theta 1} w^{2}+K_{\theta 1}\left(\frac{\partial w}{(s+a) \partial \theta}\right)^{2}\right]_{\theta=\phi}\right\} d s .
\end{aligned}
$$

The Lagrangian for the annular sector plate can be generally expressed as

$$
L=V_{p}+V_{s}-T
$$

By substituting (6) into (16) and minimizing Lagrangian against all the unknown series expansion coefficients, one is able to obtain a system of linear algebraic equations, in a matrix form, as

$$
\left(\mathbf{K}-\omega^{2} \mathbf{M}\right) \mathbf{E}=\mathbf{0}
$$

where $\mathbf{E}$ is a vector which contains all the unknown series expansion coefficients, and $\mathbf{K}$ and $\mathbf{M}$ are the stiffness and mass matrices, respectively. For conciseness, the detailed 
TABLE 1: Frequency parameters, $\Omega=\omega b^{2}(\rho h / D)^{1 / 2}$, for a completely clamped annular sector plate $(a / b=0.4, \phi=\pi / 3$, and $\mu=0.33)$.

\begin{tabular}{lcccccc}
\hline & \multicolumn{5}{c}{ Mode number } \\
& 1 & 2 & 3 & 4 & 5 & 6 \\
\hline$M=N=5$ & 85.267 & 150.14 & 194.29 & 243.66 & 266.21 & 358.17 \\
$M=N=6$ & 85.253 & 150.13 & 194.27 & 243.61 & 266.20 & 358.07 \\
$M=N=7$ & 85.257 & 150.10 & 194.23 & 243.61 & 266.07 & 358.05 \\
$M=N=8$ & 85.251 & 150.10 & 194.23 & 243.59 & 266.07 & 358.03 \\
$M=N=9$ & 85.251 & 150.10 & 194.22 & 243.59 & 266.05 & 358.03 \\
$M=N=10$ & 85.250 & 150.10 & 194.22 & 243.59 & 266.05 & 358.03 \\
$M=N=11$ & 85.250 & 150.10 & 194.22 & 243.59 & 266.04 & 358.02 \\
$M=N=12$ & 85.250 & 150.10 & 194.22 & 243.59 & 266.04 & 358.02 \\
FEM & 85.230 & 150.08 & 194.29 & 243.69 & 265.92 & 358.56 \\
Reference [22] & 85.250 & 150.10 & 194.22 & 243.59 & 266.04 & - \\
\hline
\end{tabular}

TABLE 2: Frequency parameters, $\Omega=\omega b^{2}(\rho h / D)^{1 / 2}$, for an annular sector plate with radial edges simply supported $(a / b=0.5, \phi=\pi / 4$, and $\mu=0.3)$.

\begin{tabular}{lccccccc}
\hline \multirow{2}{*}{ Circumferential edges } & \multirow{2}{*}{ Source } & \multicolumn{6}{c}{ Mode number } \\
& & 1 & 2 & 3 & 4 & 5 & 6 \\
\hline \multirow{4}{*}{ Free } & $M=N=5$ & 21.069 & 66.726 & 81.606 & 146.42 & 176.12 & 176.91 \\
& $M=N=7$ & 21.067 & 66.723 & 81.604 & 146.41 & 176.12 & 176.90 \\
& $M=N=9$ & 21.067 & 66.722 & 81.604 & 146.41 & 176.12 & 176.90 \\
& $M=N=11$ & 21.067 & 66.722 & 81.604 & 146.41 & 176.12 & 176.90 \\
& $M=N=12$ & 21.067 & 66.722 & 81.604 & 146.41 & 176.12 & 176.90 \\
& Reference [22] & 21.067 & 66.722 & 81.604 & 146.41 & 176.12 & 176.90 \\
Simply supported & Reference [30] & 21.067 & 66.722 & 81.604 & 146.41 & 176.12 & 176.90 \\
& Present & 68.379 & 150.98 & 189.60 & 278.39 & 283.59 & 387.62 \\
& Reference [22] & 68.379 & 150.98 & 189.60 & 278.39 & 283.59 & 387.62 \\
Clamped & Reference [30] & 68.379 & 150.98 & 189.60 & 278.39 & 283.59 & 387.64 \\
& Present & 107.56 & 178.82 & 269.48 & 305.84 & 346.44 & 476.29 \\
& Reference [22] & 107.57 & 178.82 & 269.49 & 305.84 & 346.46 & 476.30 \\
& Reference [30] & 107.58 & 178.82 & 269.49 & 305.84 & 346.46 & 476.30 \\
\hline
\end{tabular}

TABLE 3: Frequency parameters, $\Omega=\omega b^{2}(\rho h / D)^{1 / 2}$, for completely free annular sector plates $(a / b=0.4$ and $\mu=0.3)$.

\begin{tabular}{lccccccc}
\hline \multirow{\phi}{*}{ Source } & \multicolumn{7}{c}{ Mode number } \\
& Present & 61.515 & 2 & 3 & 4 & 5 & 6 \\
\hline \multirow{2}{*}{$\pi / 6$} & FEM & 61.516 & 67.249 & 113.97 & 149.51 & 171.91 & 244.95 \\
& Present & 15.647 & 23.576 & 38.428 & 53.649 & 63.390 & 70.739 \\
\multirow{2}{*}{$\pi / 2$} & FEM & 15.646 & 23.572 & 38.425 & 53.645 & 63.389 & 70.740 \\
\hline \multirow{2}{*}{$2 \pi / 3$} & Present & 10.148 & 16.348 & 24.588 & 35.673 & 44.649 & 59.663 \\
& FEM & 10.148 & 16.345 & 24.584 & 35.672 & 44.645 & 59.663 \\
\hline \multirow{2}{*}{$\pi$} & Present & 7.0437 & 7.6871 & 15.378 & 17.404 & 28.449 & 28.559 \\
& FEM & 7.0435 & 7.6858 & 15.374 & 17.404 & 28.445 & 28.558 \\
\hline \multirow{2}{*}{$7 \pi / 6$} & Present & 5.4291 & 6.4773 & 13.094 & 13.136 & 21.512 & 24.262 \\
& FEM & 5.4281 & 6.4769 & 13.093 & 13.131 & 21.511 & 24.258 \\
\hline \multirow{2}{*}{$3 \pi / 2$} & Present & 2.8863 & 5.2324 & 8.8762 & 9.7849 & 14.001 & 17.608 \\
& FEM & 2.8858 & 5.2320 & 8.8759 & 9.7822 & 13.999 & 17.606 \\
\hline \multirow{2}{*}{$16 \pi / 9$} & Present & 1.8048 & 3.8657 & 7.3593 & 7.7069 & 11.229 & 13.075 \\
& FEM & 1.8044 & 3.8653 & 7.3581 & 7.7066 & 11.227 & 13.074 \\
\hline \multirow{2}{*}{$2 \pi$} & Present & 1.2956 & 2.9318 & 5.7558 & 7.1511 & 9.9277 & 10.581 \\
& FEM & 1.2963 & 2.9334 & 5.7582 & 7.1519 & 9.9283 & 10.584 \\
\hline
\end{tabular}


TABLE 4: Frequency parameters, $\Omega=\omega b^{2}(\rho h / D)^{1 / 2}$, for fully clamped annular sector plates $(\mu=0.3)$.

\begin{tabular}{|c|c|c|c|c|c|c|c|c|}
\hline \multirow{2}{*}{$\phi$} & \multirow{2}{*}{$a / b$} & \multirow{2}{*}{ Source } & \multicolumn{6}{|c|}{ Mode number } \\
\hline & & & 1 & 2 & 3 & 4 & 5 & 6 \\
\hline \multirow{5}{*}{$\pi / 6$} & 0.2 & Present & 188.21 & 300.11 & 417.61 & 429.57 & 577.84 & 599.88 \\
\hline & & Present & 188.37 & 305.06 & 417.47 & 461.14 & 600.03 & 671.78 \\
\hline & 0.4 & FEM & 188.41 & 305.11 & 417.76 & 461.26 & 600.35 & 672.18 \\
\hline & & Reference [13] & 188.36 & 305.04 & 417.39 & 461.00 & 596.16 & 672.01 \\
\hline & 0.6 & Present & 216.12 & 422.67 & 454.32 & 662.94 & 728.63 & 820.91 \\
\hline \multirow{5}{*}{$\pi / 2$} & 0.2 & Present & 50.283 & 87.826 & 113.99 & 136.90 & 165.36 & 195.50 \\
\hline & & Present & 69.822 & 95.706 & 138.96 & 179.79 & 195.38 & 207.76 \\
\hline & 0.4 & FEM & 69.839 & 95.709 & 138.98 & 179.85 & 195.49 & 207.84 \\
\hline & & Reference [13] & 60.835 & 95.701 & 138.96 & 179.79 & 195.51 & 207.82 \\
\hline & 0.6 & Present & 144.27 & 159.26 & 187.24 & 228.34 & 285.34 & 351.81 \\
\hline \multirow{3}{*}{$2 \pi / 3$} & 0.2 & Present & 41.835 & 63.382 & 93.964 & 104.76 & 129.64 & 133.58 \\
\hline & 0.4 & Present & 65.700 & 78.393 & 101.37 & 133.72 & 174.32 & 175.71 \\
\hline & 0.6 & Present & 142.05 & 149.60 & 163.44 & 184.16 & 212.92 & 249.07 \\
\hline \multirow{6}{*}{$\pi$} & \multirow{2}{*}{0.2} & Present & 37.061 & 45.338 & 59.618 & 78.667 & 98.872 & 100.43 \\
\hline & & FEM & 37.043 & 45.334 & 59.767 & 78.671 & 98.962 & 100.81 \\
\hline & \multirow{2}{*}{0.4} & Present & 63.331 & 68.008 & 76.617 & 89.647 & 107.22 & 128.75 \\
\hline & & FEM & 63.329 & 68.006 & 76.606 & 89.639 & 107.14 & 128.75 \\
\hline & \multirow{2}{*}{0.6} & Present & 140.80 & 143.64 & 149.03 & 156.59 & 167.53 & 181.84 \\
\hline & & FEM & 140.64 & 143.68 & 149.98 & 156.85 & 167.62 & 181.55 \\
\hline \multirow{6}{*}{$7 \pi / 6$} & \multirow{2}{*}{0.2} & Present & 36.241 & 41.835 & 52.102 & 66.284 & 83.552 & 98.083 \\
\hline & & FEM & 36.244 & 41.844 & 52.088 & 66.314 & 83.364 & 98.114 \\
\hline & \multirow{2}{*}{0.4} & Present & 62.904 & 66.110 & 72.109 & 80.996 & 93.532 & 108.83 \\
\hline & & FEM & 62.900 & 66.142 & 72.044 & 81.035 & 93.320 & 108.84 \\
\hline & \multirow{2}{*}{0.6} & Present & 140.34 & 142.53 & 146.35 & 151.85 & 159.58 & 170.05 \\
\hline & & FEM & 140.36 & 142.53 & 146.28 & 151.78 & 159.25 & 168.88 \\
\hline \multirow{3}{*}{$3 \pi / 2$} & 0.2 & Present & 35.495 & 38.369 & 44.053 & 52.411 & 63.089 & 75.717 \\
\hline & 0.4 & Present & 62.412 & 64.212 & 67.419 & 72.312 & 79.187 & 87.876 \\
\hline & 0.6 & Present & 140.00 & 140.83 & 143.54 & 146.75 & 150.99 & 155.98 \\
\hline \multirow{3}{*}{$16 \pi / 9$} & 0.2 & Present & 35.192 & 37.153 & 40.797 & 46.446 & 54.017 & 63.212 \\
\hline & 0.4 & Present & 62.298 & 63.555 & 65.696 & 69.042 & 73.579 & 79.273 \\
\hline & 0.6 & Present & 139.94 & 141.66 & 142.46 & 144.56 & 147.48 & 151.01 \\
\hline \multirow{6}{*}{$2 \pi$} & \multirow{2}{*}{0.2} & Present & 35.061 & 36.520 & 39.252 & 43.464 & 49.307 & 56.495 \\
\hline & & FEM & 35.056 & 36.502 & 39.208 & 43.417 & 49.199 & 56.438 \\
\hline & \multirow{2}{*}{0.4} & Present & 62.188 & 63.167 & 64.820 & 67.263 & 70.707 & 75.100 \\
\hline & & FEM & 62.192 & 65.153 & 64.821 & 67.294 & 70.663 & 75.033 \\
\hline & \multirow{2}{*}{0.6} & Present & 139.72 & 140.68 & 141.75 & 143.44 & 145.58 & 148.37 \\
\hline & & FEM & 139.88 & 140.57 & 141.74 & 143.41 & 145.61 & 148.38 \\
\hline
\end{tabular}

expressions for the stiffness and mass matrices are not shown here.

The eigenvalues (or natural frequencies) and eigenvectors of annular sector plates can now be easily and directly determined from solving a standard matrix eigenvalue problem (17). For a given natural frequency, the corresponding eigenvector actually contains the series expansion coefficients which can be used to construct the physical mode shape based on (6). Although this investigation is focused on the free vibration of an annular sector plate, the response of the annular sector plate to an applied load can be easily considered by simply including the work done by this load in the Lagrangian, eventually leading to a force term on the right side of (17). Since the displacement is constructed with the same smoothness as required of a strong form of solution, other variables of interest such as shear forces and power flows can be calculated directly, and perhaps more accurately, by applying appropriate mathematical operations to the displacement function.

\section{Result and Discussion}

To demonstrate the accuracy and usefulness of the proposed technique, several numerical examples will be presented in 
TABLE 5: Frequency parameters, $\Omega=\omega b^{2}(\rho h / D)^{1 / 2}$, for CSCS annular sector plates $(\mu=0.3)$.

\begin{tabular}{|c|c|c|c|c|c|c|c|c|}
\hline \multirow{2}{*}{$\phi$} & \multirow{2}{*}{$a / b$} & \multirow{2}{*}{ Source } & \multicolumn{6}{|c|}{ Mode number } \\
\hline & & & 1 & 2 & 3 & 4 & 5 & 6 \\
\hline \multirow{7}{*}{$\pi / 4$} & \multirow{3}{*}{0.2} & Present & 70.255 & 144.22 & 168.24 & 243.43 & 282.20 & 304.07 \\
\hline & & FEM & 70.247 & 144.22 & 168.27 & 243.47 & 282.23 & 304.19 \\
\hline & & Reference [13] & 70.255 & 144.22 & 168.24 & 243.45 & 282.04 & 304.04 \\
\hline & \multirow{3}{*}{0.4} & Present & 84.594 & 169.65 & 199.19 & 296.82 & 304.11 & 366.32 \\
\hline & & FEM & 84.589 & 169.65 & 199.21 & 296.82 & 304.20 & 366.51 \\
\hline & & Reference [13] & 84.592 & 169.65 & 199.17 & 296.76 & 304.08 & 366.23 \\
\hline & 0.6 & Present & 154.74 & 211.64 & 321.79 & 404.70 & 467.22 & 481.99 \\
\hline \multirow{4}{*}{$\pi / 2$} & 0.2 & Present & 41.833 & 70.256 & 106.58 & 114.23 & 144.23 & 168.24 \\
\hline & \multirow{2}{*}{0.4} & Present & 66.678 & 86.611 & 119.45 & 169.43 & 177.64 & 199.21 \\
\hline & & FEM & 66.678 & 84.593 & 119.45 & 169.66 & 177.68 & 199.22 \\
\hline & 0.6 & Present & 143.14 & 154.76 & 176.85 & 211.65 & 259.99 & 321.76 \\
\hline \multirow{3}{*}{$2 \pi / 3$} & 0.2 & Present & 38.332 & 53.392 & 80.167 & 101.64 & 114.23 & 121.67 \\
\hline & 0.4 & Present & 64.480 & 73.627 & 91.703 & 119.46 & 155.88 & 174.66 \\
\hline & 0.6 & Present & 141.59 & 147.78 & 159.19 & 176.87 & 201.54 & 234.20 \\
\hline \multirow{6}{*}{$\pi$} & \multirow{2}{*}{0.2} & Present & 36.108 & 41.828 & 53.387 & 70.236 & 90.850 & 98.272 \\
\hline & & FEM & 36.105 & 41.820 & 53.389 & 70.255 & 90.859 & 98.294 \\
\hline & \multirow{2}{*}{0.4} & Present & 62.989 & 66.672 & 73.625 & 84.585 & 99.938 & 119.46 \\
\hline & & FEM & 63.004 & 66.678 & 73.633 & 84.597 & 99.907 & 119.46 \\
\hline & \multirow{2}{*}{0.6} & Present & 140.48 & 143.13 & 147.79 & 154.74 & 164.32 & 176.83 \\
\hline & & FEM & 140.51 & 143.17 & 147.82 & 154.76 & 164.34 & 176.87 \\
\hline \multirow{6}{*}{$7 \pi / 6$} & \multirow{2}{*}{0.2} & Present & 35.684 & 39.622 & 47.703 & 60.066 & 75.868 & 94.024 \\
\hline & & FEM & 35.684 & 39.629 & 47.699 & 60.063 & 75.821 & 94.041 \\
\hline & \multirow{2}{*}{0.4} & Present & 62.693 & 65.318 & 70.196 & 77.814 & 88.570 & 102.45 \\
\hline & & FEM & 62.700 & 65.329 & 70.199 & 77.810 & 88.526 & 102.45 \\
\hline & \multirow{2}{*}{0.6} & Present & 140.25 & 142.27 & 145.54 & 150.61 & 157.21 & 165.96 \\
\hline & & FEM & 140.29 & 142.22 & 145.56 & 150.49 & 157.22 & 165.94 \\
\hline \multirow{3}{*}{$3 \pi / 2$} & 0.2 & Present & 35.241 & 37.429 & 41.823 & 48.904 & 58.484 & 70.372 \\
\hline & 0.4 & Present & 62.367 & 63.876 & 66.619 & 70.902 & 76.824 & 84.539 \\
\hline & 0.6 & Present & 140.12 & 141.09 & 143.12 & 145.99 & 149.67 & 154.81 \\
\hline \multirow{3}{*}{$16 \pi / 9$} & 0.2 & Present & 35.036 & 36.539 & 39.462 & 44.137 & 50.791 & 59.178 \\
\hline & 0.4 & Present & 62.218 & 63.301 & 65.160 & 68.051 & 71.966 & 77.440 \\
\hline & 0.6 & Present & 139.89 & 140.63 & 142.11 & 144.11 & 146.83 & 149.99 \\
\hline \multirow{6}{*}{$2 \pi$} & \multirow{2}{*}{0.2} & Present & 34.948 & 36.119 & 38.293 & 41.824 & 46.882 & 53.362 \\
\hline & & FEM & 34.961 & 36.106 & 38.298 & 41.824 & 46.862 & 53.401 \\
\hline & \multirow{2}{*}{0.4} & Present & 62.148 & 63.172 & 64.429 & 66.683 & 69.767 & 73.579 \\
\hline & & FEM & 62.156 & 63.004 & 64.481 & 66.682 & 69.699 & 73.641 \\
\hline & \multirow{2}{*}{0.6} & Present & 139.95 & 140.33 & 141.57 & 143.13 & 145.19 & 147.67 \\
\hline & & FEM & 139.87 & 140.51 & 141.61 & 143.17 & 145.23 & 147.83 \\
\hline
\end{tabular}

this section. First, consider a completely clamped annular sector plate. A clamped B.C. can be viewed as a special case when the stiffness constants for both sets of restraining springs become infinitely large (represented by a very large number, $5.0 \times 10^{13}$, in the numerical calculations). The first six nondimensional frequency parameters, $\Omega=\omega b^{2}(\rho h / D)^{1 / 2}$, are tabulated in Table 1 together with the reference results from [22] and an FEM prediction.

Next, consider an annular sector plate with simply supported radial edges. Three different boundary conditions (free, simply supported, and clamped) are sequentially applied to the circumferential edges. The simply supported condition is simply produced by setting the stiffnesses of the translational and rotational springs to $\infty$ and 0 , respectively, and the free edge condition by setting both stiffnesses to zero. The first six nondimensional frequency parameters are shown in Table 2. The current results compare well with those taken from $[22,30]$.

To illustrate the convergence and numerical stability of the current solution, several sets of results in Tables 1 and 2 are presented for using different truncation numbers: $M=N=5,6,7, \ldots, 12$. A highly desired convergence 
TABLE 6: Frequency parameters, $\Omega=\omega b^{2}(\rho h / D)^{1 / 2}$, for simply supported annular sector plates with uniform rotational restraint along each edge $(a / b=0.4, \phi=2 \pi / 3$, and $\mu=0.3)$.

\begin{tabular}{|c|c|c|c|c|c|c|c|}
\hline \multirow{2}{*}{$K(\mathrm{Nm} / \mathrm{rad})$} & \multirow{2}{*}{ Source } & \multicolumn{6}{|c|}{ Mode number } \\
\hline & & 1 & 2 & 3 & 4 & 5 & 6 \\
\hline \multirow{2}{*}{$10^{0}$} & Present & 32.618 & 46.318 & 68.813 & 98.903 & 115.80 & 131.64 \\
\hline & FEM & 32.578 & 46.212 & 68.677 & 98.751 & 115.82 & 131.52 \\
\hline \multirow{2}{*}{$10^{4}$} & Present & 41.395 & 54.245 & 76.164 & 106.05 & 125.56 & 141.73 \\
\hline & FEM & 41.329 & 54.148 & 76.060 & 105.99 & 126.75 & 141.82 \\
\hline \multirow{2}{*}{$10^{8}$} & Present & 65.688 & 78.383 & 101.29 & 133.72 & 173.81 & 175.66 \\
\hline & FEM & 65.762 & 78.429 & 101.34 & 133.83 & 174.11 & 176.29 \\
\hline \multirow{2}{*}{$10^{12}$} & Present & 65.698 & 78.394 & 101.30 & 133.74 & 173.83 & 175.69 \\
\hline & FEM & 65.772 & 78.439 & 101.34 & 133.85 & 174.13 & 176.32 \\
\hline
\end{tabular}

TABLE 7: Frequency parameters, $\Omega=\omega b^{2}(\rho h / D)^{1 / 2}$, for an FCFF annular sector plate with identical elastic restraint at "free" edges $(a / b=$ $0.4, \phi=\pi$, and $\mu=0.3$ ).

\begin{tabular}{|c|c|c|c|c|c|c|c|}
\hline \multirow{2}{*}{$K(\mathrm{Nm} / \mathrm{rad})$} & \multirow{2}{*}{ Source } & \multicolumn{6}{|c|}{ Mode number } \\
\hline & & 1 & 2 & 3 & 4 & 5 & 6 \\
\hline \multirow{2}{*}{$10^{0}$} & Present & 4.4992 & 5.9227 & 9.6841 & 12.490 & 18.444 & 24.601 \\
\hline & FEM & 4.4898 & 5.9352 & 9.6797 & 12.491 & 18.448 & 24.576 \\
\hline \multirow{2}{*}{$10^{4}$} & Present & 4.6998 & 7.3625 & 12.924 & 20.819 & 21.912 & 30.652 \\
\hline & FEM & 4.6961 & 7.3665 & 12.926 & 20.801 & 21.938 & 30.630 \\
\hline \multirow{2}{*}{$10^{8}$} & Present & 4.7531 & 8.4457 & 15.344 & 24.898 & 30.587 & 36.816 \\
\hline & FEM & 4.7507 & 8.4435 & 15.345 & 24.909 & 30.605 & 36.842 \\
\hline \multirow{2}{*}{$10^{12}$} & Present & 4.7531 & 8.4460 & 15.345 & 24.899 & 30.589 & 36.817 \\
\hline & FEM & 4.7508 & 8.4435 & 15.346 & 24.909 & 30.606 & 36.842 \\
\hline
\end{tabular}

characteristic is observed in that (a) sufficiently accurate results can be obtained with only a small number of terms in the series expansions and (b) the solution is consistently refined as more terms are included in the expansions. While the convergence of the current solution is mathematically established via (11) and (12), the actual (truncation) error will be case-dependent and cannot be exactly determined a priori. However, this should not constitute a problem in practice because one can always verify the accuracy of the solution by increasing the truncation number until a desired numerical precision is achieved. As a matter of fact, this "quality control" scheme can be easily implemented automatically. In modal analysis, the natural frequencies for higher-order modes tend to converge slower (see Table 1). Thus, an adequate truncation number should be dictated by the desired accuracy of the largest natural frequencies of interest. In view of the excellent numerical behavior of the current solution, the truncation numbers will be simply set as $M=N=12$ in the following calculations.

In the very limited existing studies, the sector angles are typically assumed to be less than $\pi$, as specified in terms of $m=\pi / \phi$ being an integer. Although it is not clear whether $\phi=\pi$ inherently constitutes a pivoting point for mathematically solving sector plate problems, it has been a limit practically defining the previous investigations. However, the value of the sector angle appears to have no binding effect on the current solution procedures as described earlier. To verify this statement and illustrate the versatility of the proposed technique, the plates with a full range of sector angles are studied under various restraining conditions. Presented in Table 3 are the first six frequency parameters, $\Omega=\omega b^{2}(\rho h / D)^{1 / 2}$, for annular sector plates $(a / b=0.4)$ which are completely free along all of their edges. Due to a lack of analytical solutions, the numerical results calculated using an FEM (ABAQUS) model are given there for comparison. Since the reference solutions for annular sector plates are not readily available, the plates with other classical boundary conditions are also studied systematically and the corresponding results are listed in Tables 4 and 5 for a range of sector angles up to $2 \pi$. Such results can be particularly useful in benchmarking other solution methods. In identifying the boundary conditions, letters $\mathrm{C}, \mathrm{S}$, and $\mathrm{F}$ have been used to indicate the clamped, simply supported, and free boundary condition along an edge, respectively. Thus, the boundary conditions for a plate are fully specified by using four letters with the first one indicating the B.C. along the first edge, $r=a$. The remaining (the second to the fourth) edges are ordered in the counterclockwise direction. In all these cases, the current solutions are adequately validated by the FEM results obtained using ABAQUS models. Also included are the results previously given in [13] for smaller sector angles, $\phi=\pi / 6$ and $\pi / 2$. The mode shapes for the first six modes are plotted in Figure 2 for the fully clamped annular sector plate with cutout ratio $a / b=0.4$ and sector angle $\phi=\pi$. These modes are verified by the FEM results although they will not be shown here for conciseness. 
TABLE 8: Frequency parameters, $\Omega=\omega b^{2}(\rho h / D)^{1 / 2}$, for annular sector plates with elastic restraint at all four edges: $k=10^{5} \mathrm{~N} / \mathrm{m}$ and $K=$ $10^{7} \mathrm{Nm} / \mathrm{rad}(\phi=16 \pi / 9$ and $\mu=0.3)$.

\begin{tabular}{|c|c|c|c|c|c|c|c|}
\hline \multirow{2}{*}{$a / b$} & \multirow{2}{*}{ Source } & \multicolumn{6}{|c|}{ Mode number } \\
\hline & & 1 & 2 & 3 & 4 & 5 & 6 \\
\hline \multirow{2}{*}{0.2} & Present & 10.268 & 11.702 & 13.324 & 15.209 & 17.664 & 21.015 \\
\hline & FEM & 10.251 & 11.684 & 13.311 & 15.209 & 17.678 & 21.038 \\
\hline \multirow{2}{*}{0.4} & Present & 11.884 & 12.593 & 13.802 & 15.471 & 17.763 & 20.988 \\
\hline & FEM & 11.862 & 12.575 & 13.795 & 15.477 & 17.780 & 21.009 \\
\hline \multirow{2}{*}{0.6} & Present & 14.586 & 14.934 & 15.663 & 16.794 & 18.413 & 20.849 \\
\hline & FEM & 14.557 & 14.908 & 15.650 & 16.802 & 18.441 & 20.884 \\
\hline
\end{tabular}

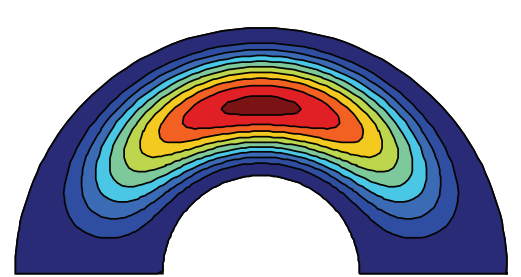

(a)

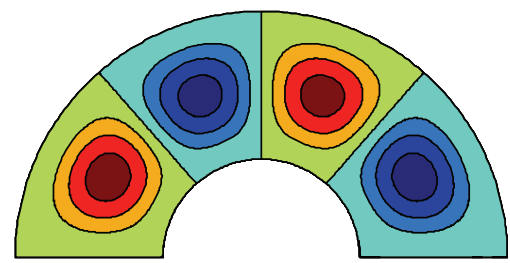

(d)

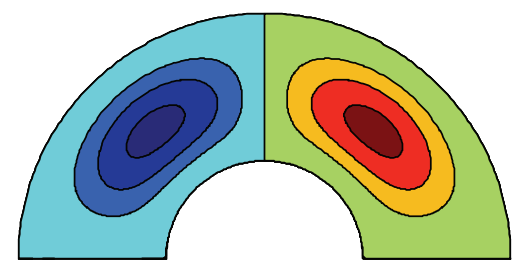

(b)

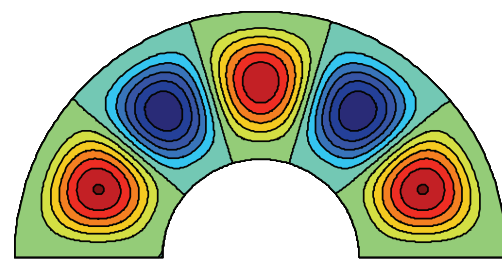

(e)

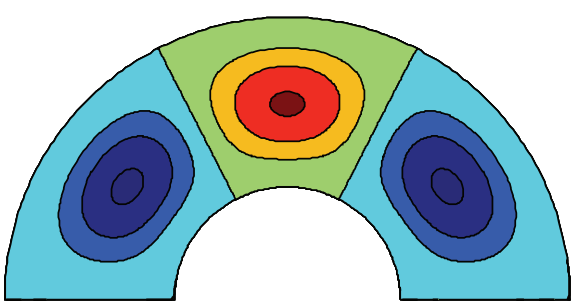

(c)

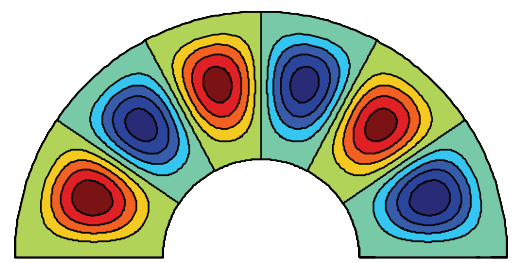

(f)

FIgurE 2: The first six mode shapes for a CCCC annular sector plate $(a / b=0.4$ and $\phi=\pi)$ : the (a) first, (b) second, (c) third, (d) fourth, (e) fifth, and (f) sixth mode shape.

All the above examples involve the classical homogeneous boundary conditions which are viewed as special cases (of elastically restrained edges) when the stiffness constants take extreme values. We now turn to annular sector plates with general elastically restrained edges. First consider an annular sector plate simply supported, but with uniform rotational restraint, along each edge. The first six frequency parameters are presented in Table 6 together with the results calculated using an ABAQUS model. The second example concerns a cantilever annular sector plate (clamped at $\theta=0$ ) with identical elastic restraints at other edges. While the stiffness of the translational springs is fixed to $k=10^{4} \mathrm{~N} / \mathrm{m}$, the rotational springs will be specified to take different stiffness values: $K=10^{0}, 10^{4}, 10^{8}, 10^{12} \mathrm{Nm} / \mathrm{rad}$. The corresponding frequency parameters are shown in Table 7 . In all the cases, a good agreement is observed between the current solution and the FEM results.

Lastly, consider reentrant annular sector plates $(\phi=$ $16 \pi / 9$ ) elastically restrained along all the four edges. The stiffnesses for the translational and rotational restraints is chosen as $k=10^{5} \mathrm{~N} / \mathrm{m}$ and $K=10^{7} \mathrm{Nm} / \mathrm{rad}$, respectively. The first six frequency parameters are shown in Table 8 for three different cutout ratios. Plotted in Figure 3 are the mode shapes for the plate with $a / b=$ 0.4 .

\section{Conclusions}

An analytical method has been presented for the vibration analysis of annular sector plates with general elastic restraints along each edge, which allows treating all the classical homogenous boundary conditions as the special cases when the stiffness for each of the restraining spring is equal to either zero or infinity. Regardless of boundary conditions, the displacement function is invariantly expressed as an improved trigonometric series which converges uniformly at an accelerated rate. Since the displacement solution is constructed to have $C^{3}$ continuity, the current solution, although sought in a weak form from the Rayleigh-Ritz procedure, is mathematically equivalent to a strong solution which simultaneously satisfies both the governing differential equation and the boundary conditions on a point-wise basis.

The present method provides a unified means for predicting the free vibration characteristics of annular sector plates with a variety of boundary conditions and any sector angles. The efficiency, accuracy, and reliability of the proposed method are fully illustrated for free vibration analysis of annular sector plates with different boundary supports and model parameters such as radius ratio and sector angle. Numerical results obtained by the present approach are in excellent agreement with those available in the literature. 


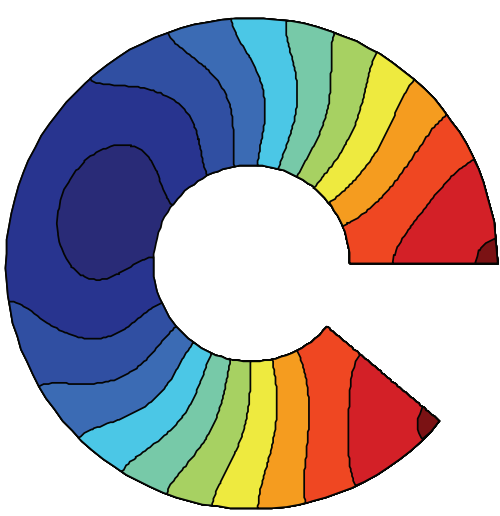

(a)

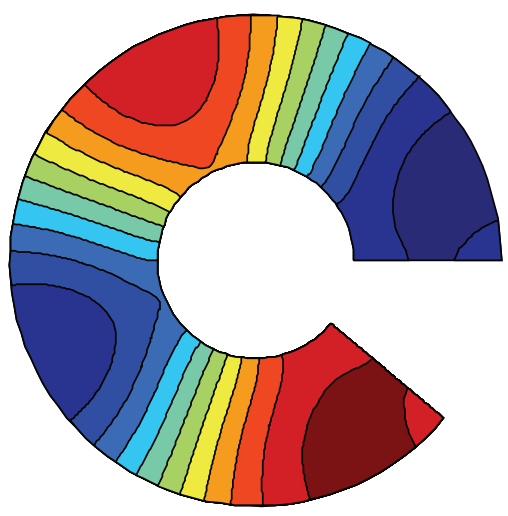

(d)

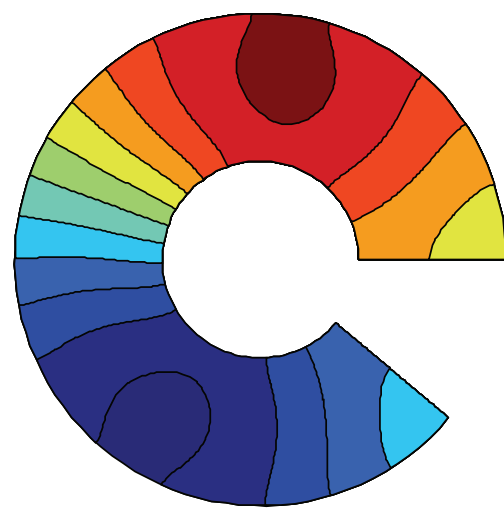

(b)

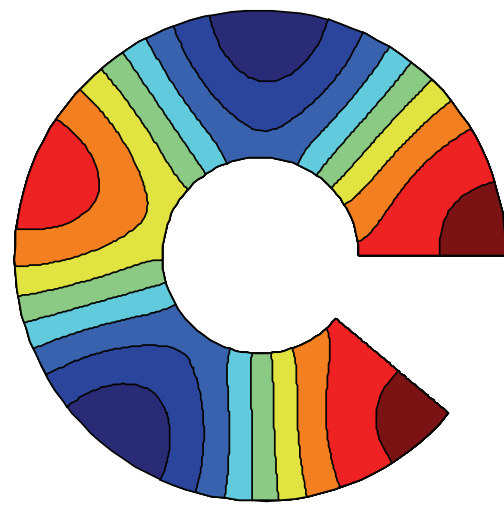

(e)

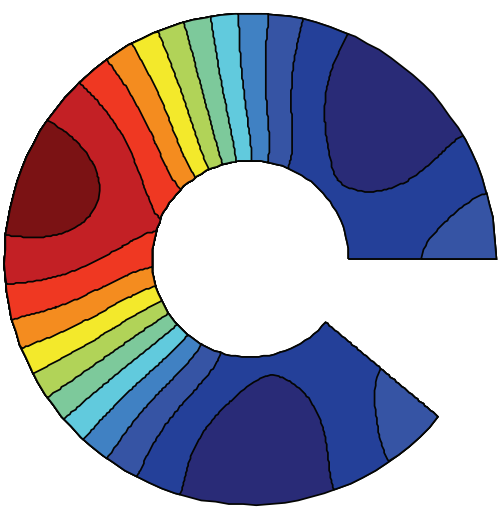

(c)

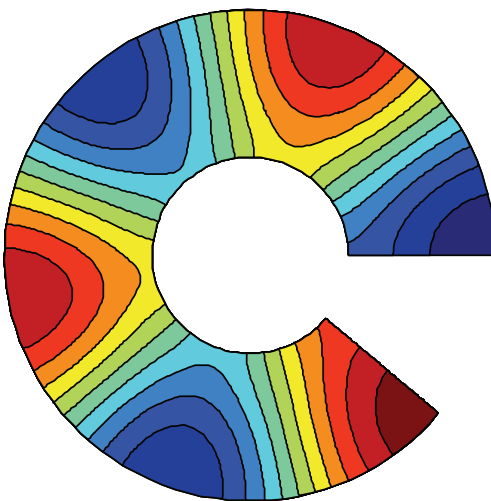

(f)

FIGURE 3: The first six mode shapes for an annular sector plate $(a / b=0.4$ and $\phi=16 \pi / 9)$ with elastic restraints, $k=10^{5} \mathrm{~N} / \mathrm{m}$ and $K=$ $10^{7} \mathrm{Nm} / \mathrm{rad}$, at all the four edges: the (a) first, (b) second, (c) third, (d) fourth, (e) fifth, and (f) sixth mode shape.

Although the stiffness for each restraining spring is here assumed to be uniform, any nonuniform, discrete, or partial stiffness distribution can be readily considered by modifying potential energies accordingly.

\section{Conflict of Interests}

The authors declare that there is no conflict of interests regarding the publication of this paper.

\section{Acknowledgments}

The authors would like to thank the anonymous reviewers for their very valuable comments. This work was supported by the International S\&T Cooperation Program of China (2011DFR90440) and the key project of the National Natural Science of Foundation of China (50939002). The second author is also grateful for the supports from China Scholarship Council (2011668004).

\section{References}

[1] A. W. Leissa, Vibration of Plates, U. S. Government Printing Office, Washington DC, USA, 1969.
[2] G. K. Ramaiah and K. Vijayakumar, "Natural frequencies of circumferentially truncated sector plates with simply supported straight edges," Journal of Sound and Vibration, vol. 34, no. 1, pp. 53-61, 1974.

[3] T. Mizusawa and H. Ushijima, "Vibration of annular sector Mindlin plates with intermediate arc supports by the spline strip method," Computers and Structures, vol. 61, no. 5, pp. 819-829, 1996.

[4] T. Mizusawa, H. Kito, and T. Kajita, "Vibration of annular sector mindlin plates by the spline strip method," Computers and Structures, vol. 53, no. 5, pp. 1205-1215, 1994.

[5] M. N. Bapu Rao, P. Guruswamy, and K. S. Sampathkumaran, "Finite element analysis of thick annular and sector plates," Nuclear Engineering and Design, vol. 41, no. 2, pp. 247-255, 1977.

[6] R. S. Srinivasan and V. Thiruvenkatachari, "Free vibration of annular sector plates by an integral equation technique," Journal of Sound and Vibration, vol. 89, no. 3, pp. 425-432, 1983.

[7] A. Houmat, "A sector Fourier p-element applied to free vibration analysis of sectorial plates," Journal of Sound and Vibration, vol. 243, no. 2, pp. 269-282, 2001.

[8] A. W. Leissa, O. G. McGee, and C. S. Huang, "Vibrations of sectorial plates having corner stress singularities," Journal of Applied Mechanics, vol. 60, no. 1, pp. 134-140, 1993.

[9] K. M. Liew, Y. Xiang, and S. Kitipornchai, "Research on thick plate vibration: a literature survey," Journal of Sound and Vibration, vol. 180, no. 1, pp. 163-176, 1995. 
[10] K. Kim and C. H. Yoo, "Analytical solution to flexural responses of annular sector thin-plates," Thin-Walled Structures, vol. 48, no. 12, pp. 879-887, 2010.

[11] R. Ramakrishnan and V. X. Kunukkasseril, "Free vibration of annular sector plates," Journal of Sound and Vibration, vol. 30, no. 1, pp. 127-129, 1973.

[12] M. M. Aghdam, M. Mohammadi, and V. Erfanian, "Bending analysis of thin annular sector plates using extended Kantorovich method," Thin-Walled Structures, vol. 45, no. 12, pp. 983-990, 2007.

[13] Y. Xiang, K. M. Liew, and S. Kitipornchai, "Transverse vibration of thick annular sector plates," Journal of Engineering Mechanics, vol. 119, no. 8, pp. 1579-1599, 1993.

[14] C. F. Liu and G. T. Chen, "A simple finite element analysis of axisymmetric vibration of annular and circular plates," International Journal of Mechanical Sciences, vol. 37, no. 8, pp. 861-871, 1995.

[15] Ö. Civalek and M. Ülker, "Harmonic differential quadrature (HDQ) for axisymmetric bending analysis of thin isotropic circular plates," Structural Engineering and Mechanics, vol. 17, no. 1, pp. 1-14, 2004.

[16] Ö. Civalek, "Application of differential quadrature (DQ) and harmonic differential quadrature (HDQ) for buckling analysis of thin isotropic plates and elastic columns," Engineering Structures, vol. 26, no. 2, pp. 171-186, 2004.

[17] K. M. Liew, T. Y. Ng, and B. P. Wang, "Vibration of annular sector plates from three-dimensional analysis," Journal of the Acoustical Society of America, vol. 110, no. 1, pp. 233-242, 2001.

[18] X. Wang and Y. Wang, "Free vibration analyses of thin sector plates by the new version of differential quadrature method," Computer Methods in Applied Mechanics and Engineering, vol. 193, no. 36-38, pp. 3957-3971, 2004.

[19] T. Irie, G. Yamada, and F. Ito, "Free vibration of polarorthotropic sector plates," Journal of Sound and Vibration, vol. 67, no. 1, pp. 89-100, 1979.

[20] D. Zhou, S. H. Lo, and Y. K. Cheung, "3-D vibration analysis of annular sector plates using the Chebyshev-Ritz method," Journal of Sound and Vibration, vol. 320, no. 1-2, pp. 421-437, 2009.

[21] A. H. Baferani, A. R. Saidi, and E. Jomehzadeh, "Exact analytical solution for free vibration of functionally graded thin annular sector plates resting on elastic foundation," Journal of Vibration and Control, vol. 18, no. 2, pp. 246-267, 2012.

[22] S. H. Mirtalaie and M. A. Hajabasi, "Free vibration analysis of functionally graded thin annular sector plates using the differential quadrature method," Proceedings of the Institution of Mechanical Engineers C: Journal of Mechanical Engineering Science, vol. 225, no. 3, pp. 568-583, 2011.

[23] E. Jomehzadeh and A. R. Saidi, "Analytical solution for free vibration of transversely isotropic sector plates using a boundary layer function," Thin-Walled Structures, vol. 47, no. 1, pp. 8288, 2009.

[24] W. L. Li, "Free vibrations of beams with general boundary conditions," Journal of Sound and Vibration, vol. 237, no. 4, pp. 709-725, 2000.

[25] W. L. Li, M. W. Bonilha, and J. Xiao, "Vibrations of two beams elastically coupled together at an arbitrary angle," Acta Mechanica Solida Sinica, vol. 25, no. 1, pp. 61-72, 2012.

[26] W. L. Li, "Vibration analysis of rectangular plates with general elastic boundary supports," Journal of Sound and Vibration, vol. 273, no. 3, pp. 619-635, 2004.
[27] W. L. Li, X. Zhang, J. Du, and Z. Liu, "An exact series solution for the transverse vibration of rectangular plates with general elastic boundary supports," Journal of Sound and Vibration, vol. 321, no. 1-2, pp. 254-269, 2009.

[28] D. Y. Shi, X. J. Shi, W. L. Li, and Q. S. Wang, "Free transverse vibrations of orthotropic thin rectangular plates with arbitrary elastic edge supports," Journal of Vibroengineering, vol. 16, no. 1, pp. 389-398, 2014.

[29] X. Zhang and W. L. Li, "Vibrations of rectangular plates with arbitrary non-uniform elastic edge restraints," Journal of Sound and Vibration, vol. 326, no. 1-2, pp. 221-234, 2009.

[30] C. S. Kim and S. M. Dickinson, "On the free, transverse vibration of annular and circular, thin, sectorial plates subject to certain complicating effects," Journal of Sound and Vibration, vol. 134 , no. 3, pp. 407-421, 1989. 

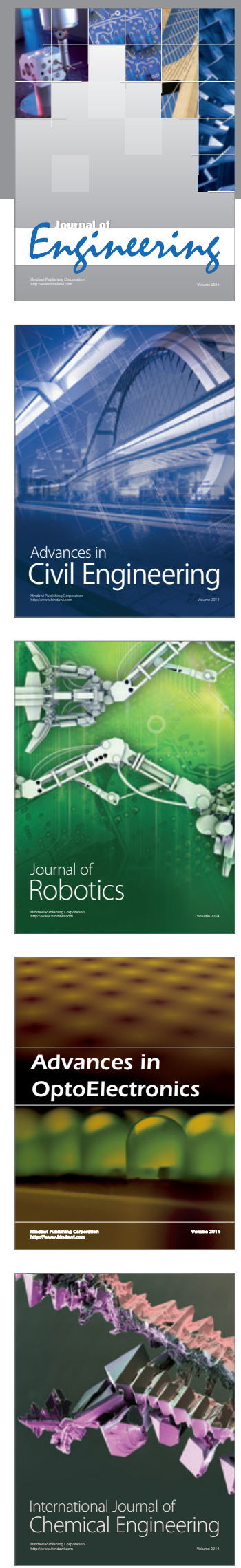

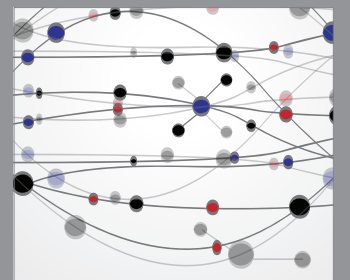

The Scientific World Journal
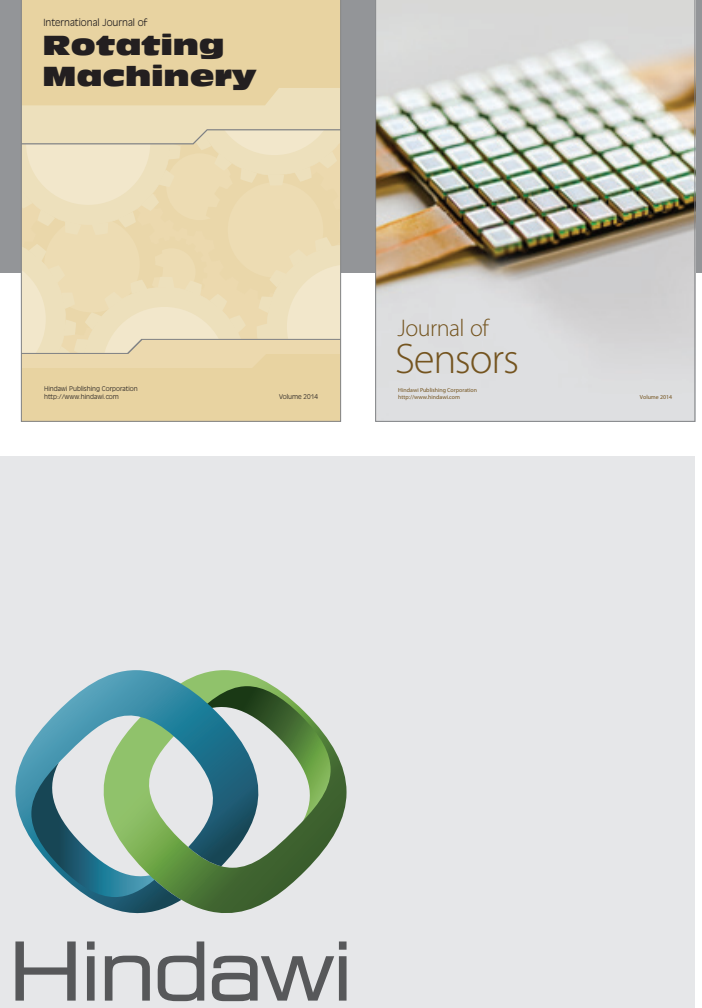

Submit your manuscripts at http://www.hindawi.com
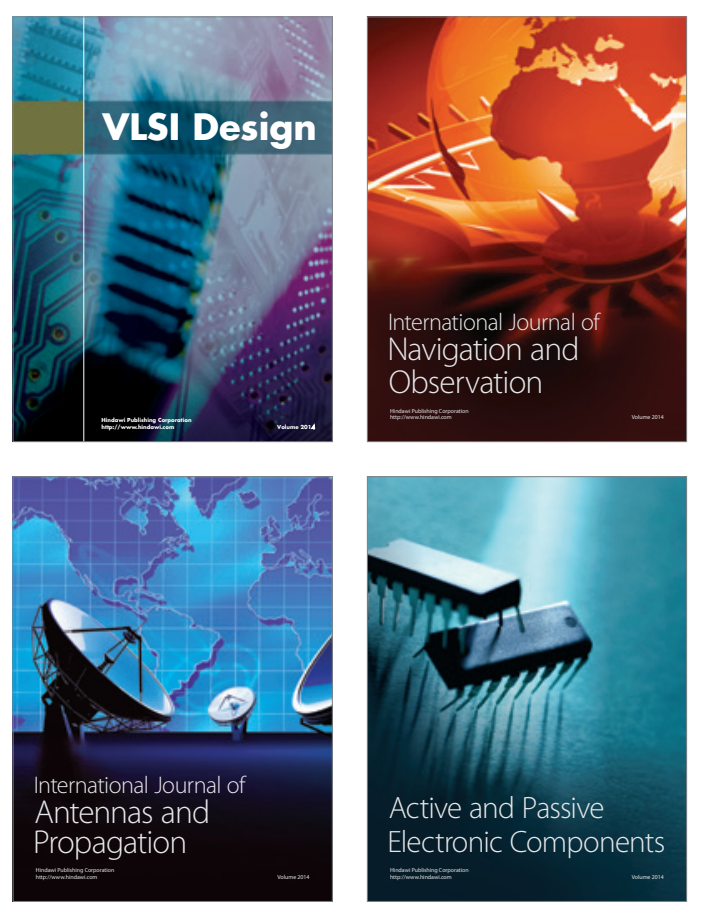
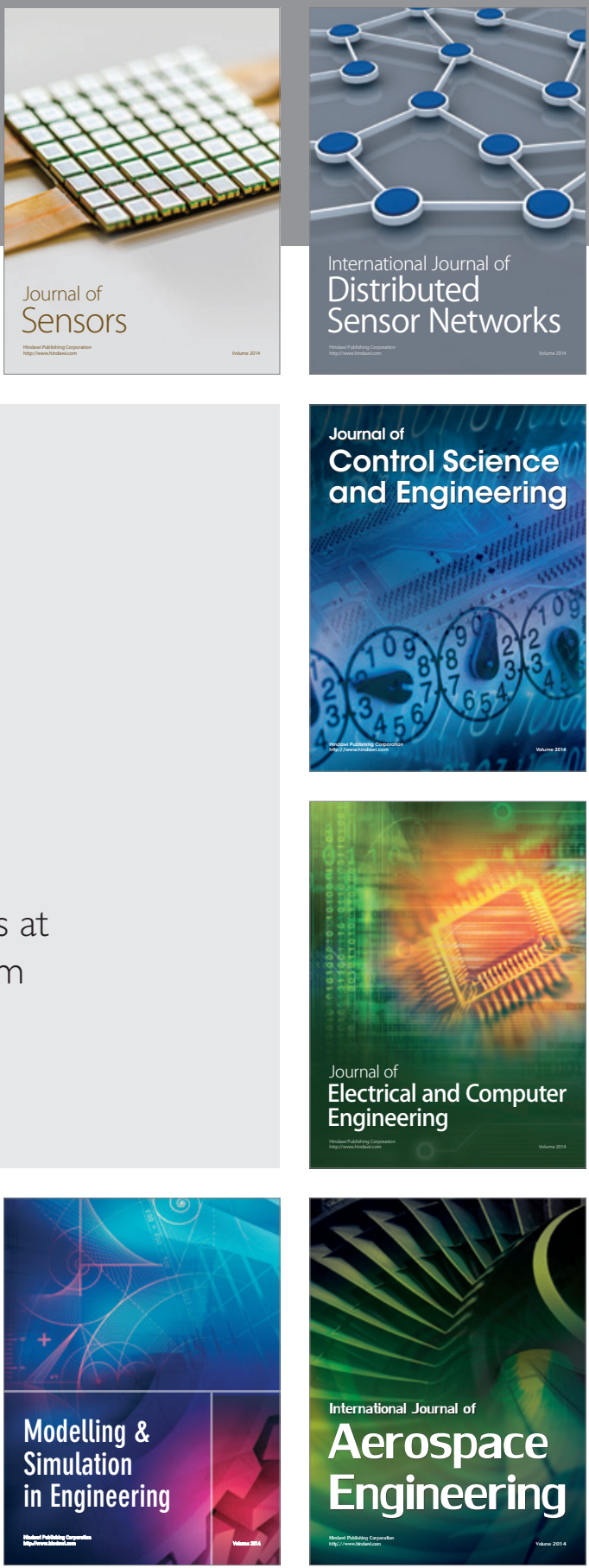

Journal of

Control Science

and Engineering
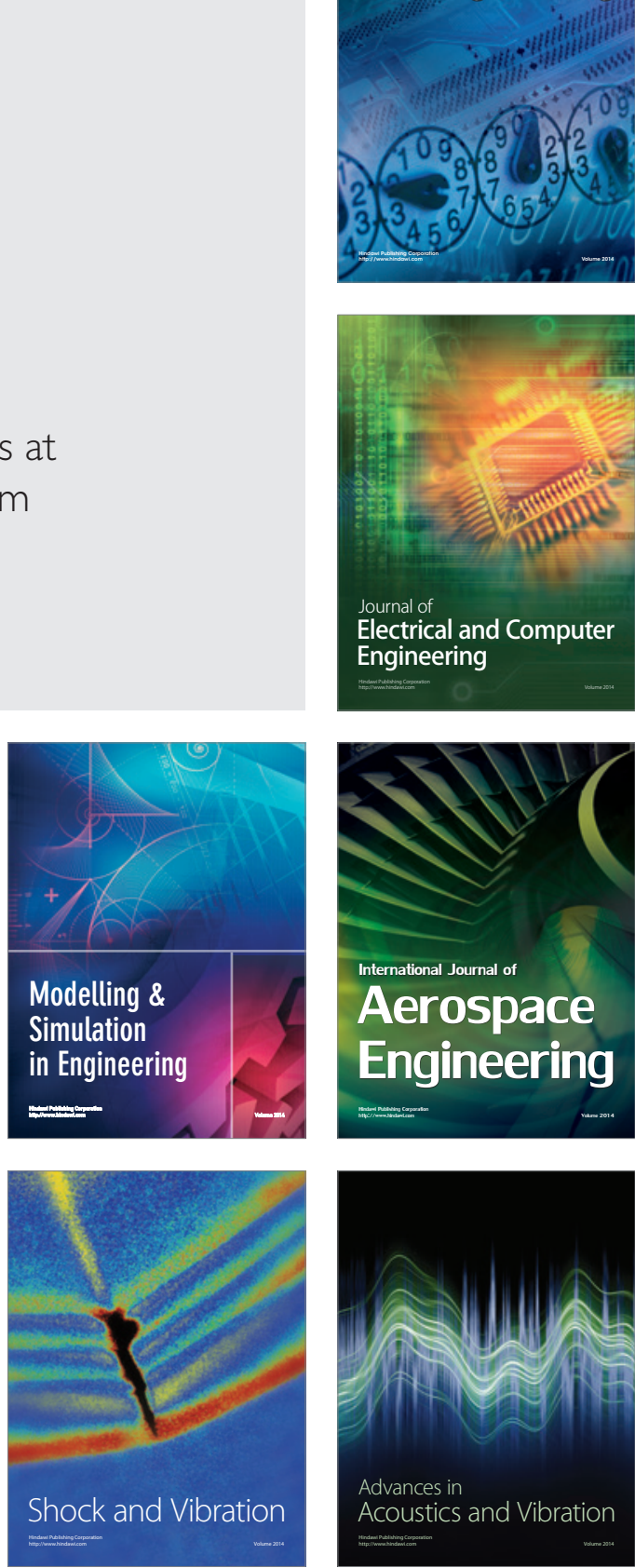